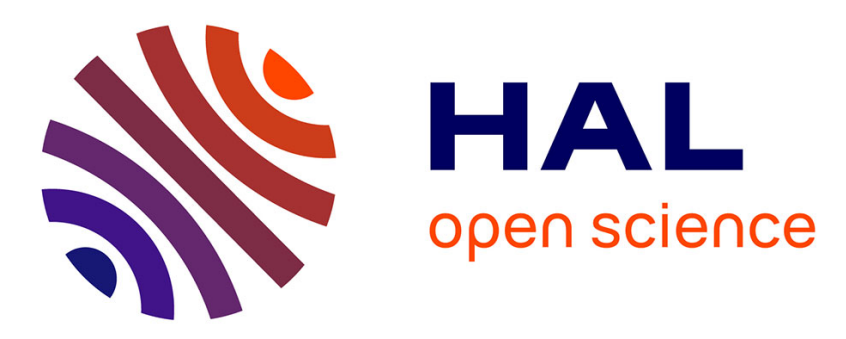

\title{
Une cavité résonnante centimétrique pour l'excitation des gaz
}

\author{
Christian Dupret, Bernard Vidal, Pierre Goudmand
}

\section{To cite this version:}

Christian Dupret, Bernard Vidal, Pierre Goudmand. Une cavité résonnante centimétrique pour l'excitation des gaz. Revue de Physique Appliquée, 1970, 5 (2), pp.337-338. 10.1051/rphysap:0197000502033700 . jpa-00243398

\section{HAL Id: jpa-00243398 https://hal.science/jpa-00243398}

Submitted on 1 Jan 1970

HAL is a multi-disciplinary open access archive for the deposit and dissemination of scientific research documents, whether they are published or not. The documents may come from teaching and research institutions in France or abroad, or from public or private research centers.
L'archive ouverte pluridisciplinaire HAL, est destinée au dépôt et à la diffusion de documents scientifiques de niveau recherche, publiés ou non, émanant des établissements d'enseignement et de recherche français ou étrangers, des laboratoires publics ou privés. 


\title{
UNE CAVITÉ RÉSONNANTE CENTIMÉTRIQUE POUR L'EXCITATION DES GAZ
}

\author{
par MM. Christian DUPRET, Bernard VIDAL et Pierre GOUDMAND
}

(Reçu le 22 décembre 1969)

\begin{abstract}
Résumé. - Nous décrivons une cavité résonnante coaxiale fonctionnant sur la bande des $2450 \mathrm{Mc} / \mathrm{s}$ qui permet l'excitation des gaz dans un large domaine de pressions.

Abstract. - A simple microwave cavity, operating at $2450 \mathrm{MHz}$, for producing discharges in gases, is tested in $\mathrm{N}_{2}, \mathrm{Ar}$ and $\mathrm{H}_{2}$ at pressures from $3 \cdot 10^{-5} \mathrm{~mm} \mathrm{Hg}$ to one atmosphere.
\end{abstract}

L'emploi d'ondes situées dans le domaine des hyperfréquences (bande légale des $2450 \mathrm{Mc} / \mathrm{s}$ ) pour l'excitation des gaz, en régime dynamique ou statique, s'est largement répandu depuis quelques années [1] [2]. Les cavités résonnantes utilisées transmettent la puissance haute fréquence aux gaz avec une grande efficacité tout en évitant les inconvénients présentés par les tubes à décharge classiques (hautes tensions, impuretés dues aux électrodes).

La plupart des cavités existant actuellement ont été calculées par H. P. Broida [2] et construites d'après ses plans. Nous avons voulu étudier une cavité dans laquelle l'arrivée du gaz par l'intérieur même du piston d'accord permettrait au gaz excité (plasma) de participer à sa propre excitation en facilitant la conduction des ondes de l'antenne vers le piston. Nous pouvions de ce fait espérer un plus haut rendement en espèces activées.

Nous décrivons dans ce travail une cavité coaxiale dont la fréquence de résonance est ajustée par un piston mobile creux. Le couplage est effectué au moyen d'une antenne de longueur variable située à $\lambda / 4$ du fond de la cavité (schéma). L'ensemble est prévu pour être traversé par des tubes de quartz de diamètre maximum $13 \mathrm{~mm}$ dans lesquels circule le gaz subissant la décharge. Le refroidissement de cette dernière est effectué par une arrivée d'air comprimé au niveau de l'antenne et perpendiculairement à celle-ci (Fig. 1 et 2).

Le réglage de l'antenne est commandé de l'extérieur par une tige de téflon solidaire d'une partie métallique mobile qui se visse plus ou moins sur une tige fixe.

Une bague de téflon dans l'espace de liaison prisecavité assure un bon isolement des différents conducteurs. L'âme centrale du câble coaxial peut être éventuellement soudée à la partie fixe de l'antenne.

L'ensemble de la cavité est construit en laiton rigide. Un bon contact doit être maintenu entre le piston et la cavité si l'on veut éviter des microfusions par étincelles à ce niveau qui nuiraient au bon rendement de

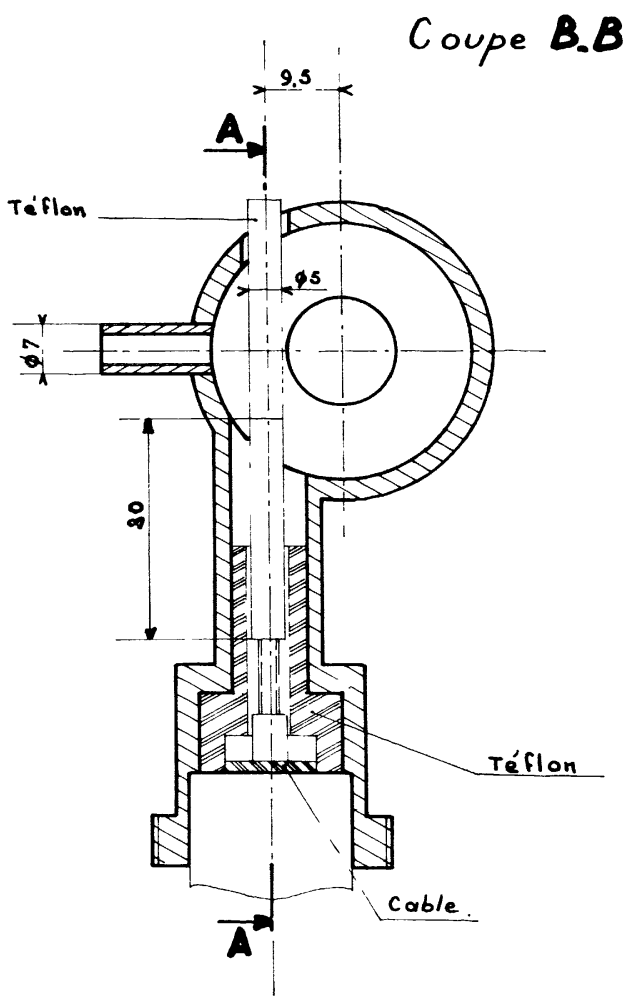

Fig. 1. - Coupe de la cavité selon B. B.

l'ensemble. Pour améliorer l'homogénéité du contact nous avons déposé par chauffage une pellicule d'un alliage à base d'argent (soudaline) à l'intérieur du trou de passage du piston.

Le passage du gaz dans l'axe de la cavité permet d'utiliser plus efficacement le transfert d'énergie antenne-cavité par l'intermédiaire du gaz excité. Nous avons ainsi pu obtenir les résultats consignés dans le tableau suivant en testant la cavité à partir des trois gaz $\mathrm{H}_{2}, \mathrm{~N}_{2}$, Ar de $3 \times 10^{-5} \mathrm{~mm} \mathrm{Hg}$ jusqu'à des pressions de l'ordre de l'atmosphère. Dans le domaine des basses pressions nous avons été limité à $3 \times 10^{-5} \mathrm{~mm}$ 


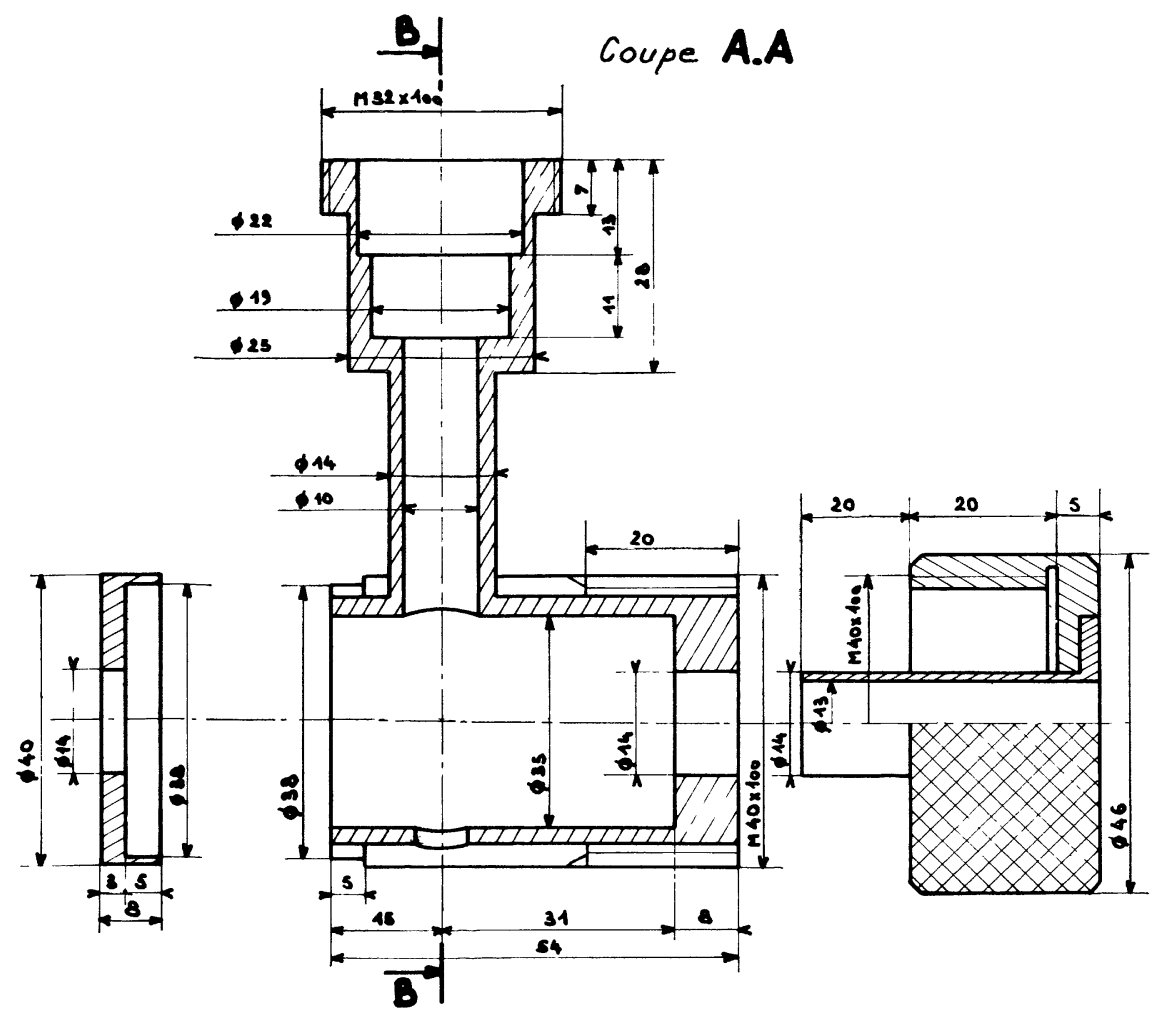

FIG. 2. - Coupe de la cavité selon A. A.

$\mathrm{Hg}$ par les caractéristiques du système de pompage, même à cette pression les flammes conservent toute leur stabilité et leur luminosité.

Au-dessus de $230 \mathrm{~mm} \mathrm{Hg}$ d'azote un arc se produit entre le piston et l'antenne, certainement dû au fait que le gaz excité est pratiquement de même nature que le gaz à l'intérieur de la cavité.

Pour l'hydrogène la limite vers les hautes pressions provient du fait que le refroidissement utilisé ne permet pas d'empêcher la fusion du quartz. L'excitation de l'argon peut, par contre, se poursuivre jusqu'à des pressions supérieures à la pression atmosphérique.

Domaine de fonctionnement

$\begin{array}{lll} & \mathrm{P} \text { Minima } & \mathrm{P} \text { Maxima } \\ \mathrm{H}_{2} & <3 \times 10^{-5} \mathrm{~mm} \mathrm{Hg} & >640 \mathrm{~mm} \mathrm{Hg} \\ \mathrm{N}_{2} & <3 \times 10^{-5} & 230 \\ \mathrm{Ar} & <3 \times 10^{-5} & >990\end{array}$

Ce travail a été réalisé avec la collaboration technique de Alain Gillon.

\section{Bibliographie}

[1] Peyron (M.), J. Chim. Phys., 1962, 59, 99.

[2] Fehsenfeld (F. C.), Evenson (K. M.) et Broida (H. P.), N. B. S., Report 8701, 1964. 